\title{
IEEE 802.3 Ethernet, Current Status and Future Prospects at the LHC
}

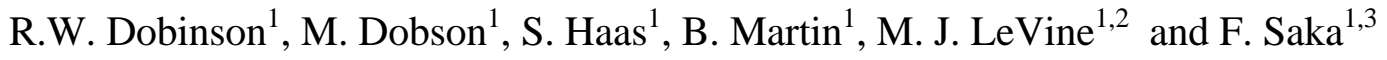 \\ ${ }^{1}$ CERN, 1211 Geneva 23, Switzerland \\ ${ }^{2}$ Brookhaven National Laboratory, Upton, NY 11973-5000, USA \\ ${ }^{3}$ Royal Holloway College, University of London, Egham, UK
}

\begin{abstract}
The status of the IEEE 802.3 standard is reviewed and prospects for the future, including the new 10 Gigabit version of Ethernet, are discussed. The relevance of Ethernet for experiments at the CERN Large Hadron Collider is considered, with emphasis on on-line applications and areas which are technically challenging.
\end{abstract}

\section{INTRODUCTION}

Ethernet was conceived and implemented in the research laboratories of Xerox in 1973 and described in the classic paper of Metcalfe and Boggs in 1976 [1]. Nodes on this local area network (LAN) communicated over a shared coaxial cable at a speed of $3 \mathrm{Mbps}$. It used the now famous CSMA-CD scheme to make available the shared bandwidth of the cable to multiple network nodes.

In 1979 DEC and Xerox joined forces to commercialize products based on Ethernet technology. Intel was added to the consortium to provide silicon know how. This turned out to be a far sighted move by the other two partners as low cost silicon has been one of the keys to the success of Ethernet. The availability of cheap electronic and photonic components is what will determine the pace of progress to ever higher speed Ethernet components and systems.

The original DIX standard ran at a single speed of $10 \mathrm{Mbps}$ over a thick multi-drop coaxial cable. It defined the Ethernet frame format, address fields and minimum/maximum frame lengths, as well as the details of the CSMA-CD media access control (MAC) layer. The frame structure has remained essentially unchanged over the last twenty years.

The frame format may not have changed in the intervening years, but in other ways Ethernet has evolved dramatically from its original starting point. This paper reviews the changes which have lead to the present IEEE 802.3 standard [2]. It focuses attention on the new ten Gigabit version of Ethernet and looks at prospects for use of the standard at the LHC, in particular for on-line applications. The likely technical challenges are identified.

\section{FORMAL STANDARDIZATION AND CONSOLIDATION}

In 1980, in parallel with the DIX work, the IEEE set up its project 802 as an industry platform for standardizing LAN technology. Agreeing to a single LAN standard proved impossible and the result was not one standard but three, the other two being IEEE 802.4 Token Bus and IEEE 802.5 Token Ring. IEEE 802.3 Ethernet based very largely on the DIX work, was standardized in 1983. However, in addition to the 10 Mbps coaxial cable version, a 1 Mbps CSMA-CD system was designated for use over twisted pair cabling. The 1
Mbps speed never caught on, but the use of CSMA-CD over twisted pair was picked up later on in the evolution of Ethernet.

It is interesting to note that the maximum speed of the Ethernet standard remained at $10 \mathrm{Mbps}$ until 1995. There were however significant developments in two areas, the physical medium over which the communication took place and the area of bridging and switching.

The original thick Ethernet cable proved somewhat unwieldy and costly to install and operate; it was therefore supplemented by a new "Cheapernet" version of the standard running over thin coaxial cable in 1985. Cheapernet had a reduced distance span, $185 \mathrm{~m}$ compared to $500 \mathrm{~m}$, without repeaters. However, it had significant advantages in terms of compactness and allowed all components needed to connect a host computer to Ethernet to be mounted on a single, small and cheap network interface card.

The widespread use of structured cabling in buildings lead to the adoption of a version of 802.3 running over unshielded twisted pair in 1990. This has become the dominant medium over which Ethernet frames are transmitted. The original physical shared cable topology is effectively emulated using the star-wired topology of structured cabling, with hubs at the centre of the star. A standard for $10 \mathrm{Mbps}$ Ethernet over multimode optical fibre was introduced in 1993.

The successful operation of CSMA-CD imposes a restriction on the length of an Ethernet segment, or collision domain, dependent on the minimum size packet and the transmission speed over the transmission medium. In order to detect collisions, the round trip time on a segment, in the conceptually simplest case a length of coaxial cable, must be less than the time slot corresponding to the smallest size packet that can be transmitted. The minimum size frame is specified to be 64 bytes, hence at $10 \mathrm{Mbps}$ the maximum distance between two nodes is about $2.5 \mathrm{~km}$, based on the fundamental limitation imposed by the collision detection window.

\section{BRIDGES AND SWITCHES}

One of the reasons why the maximum speed of Ethernet remained static for so many years was the enhancement of the original architecture by the addition of bridges and switches. A bridge is a transparent device connecting two Ethernet segments. Each port examines Ethernet frames generated on its local segment and passes those with addresses it does not recognize as local across the bridge and onto the far segment. The two segments can operate completely independently in parallel as long as the traffic is local, thus effectively increasing the bandwidth of the network by a factor two. 
Multi-segment networks increase still further the total volume of traffic that can be carried, as well as the distance spanned.

The first bridge was introduced by DEC in 1983. Early bridges, although they had low performance, became very popular devices for building campus networks and were offered by a number of manufacturers. The performance limitations were not usually a problem given the local nature of LAN traffic at that time. Work to allow vendor interoperability resulted in an IEEE bridge standard in 1990.

In the early 90's a new class of multi-port bridge appeared on the scene supporting a large number of network nodes on multiple segments and offering full Ethernet line speed. These devices became known as LAN switches. They were increasingly used for dedicated connections (a single node on a segment) when high performance was required. This was the first step in removing collisions from Ethernet. A dedicated segment is no longer a shared medium, there are no collisions and the full bandwidth is available to a single node. The segment becomes a point to point link which can support full duplex operation instead of the original half duplex. In addition the distance limitations of CSMA-CD are removed. Full duplex operation of Ethernet was standardized in 1997 along with a standard method for exerting back pressure using flow control frames.

\section{FAST AND GIGABIT ETHERNET}

The use of switches brought a full $10 \mathrm{Mbps}$ capability onto the user's desk top, this fuelled the need for higher speed backbones and server connections. This requirement resulted in the 100 Mbps Fast Ethernet standard in 1995. This higher speed version of Ethernet has basically the same characteristics as the $10 \mathrm{Mbps}$ standard in terms of frame format, size and CSMA-CD media access control. However, it has a reduced distance coverage for a collision domain, compared to 10 Mbps, due to its higher speed. There is no impact on software interfaces at the user level, except of course for the increase in performance. Both twisted pair and optical fibre transmission media are supported.

Fast Ethernet proved to be an enormous success, spawning a wave of new high performance Ethernet products. In particular, it encouraged the further move away from shared segments to point to point, full duplex links and switches.

Although the time lag between $10 \mathrm{Mbps}$ and $100 \mathrm{Mbps}$ Ethernet was 15 years, there was a rapid move towards even higher speed once the Fast Ethernet standard was in place. The combination of $100 \mathrm{Mbps}$ and switching required an evolution to $1 \mathrm{Gbps}$ and an IEEE task force was formed in 1996 to develop such a standard.

The Gigabit Ethernet standard, approved in 1998, once again retained the basic characteristics of the original $10 \mathrm{Mbps}$ version in terms of frame format and provision of CSMA-CD. The initial version ran over optical fibre and shielded twisted pair (twinax). In practice neither the CSMA-CD option nor the shielded twisted pair transmission medium have turned out to be popular with LAN equipment manufacturers. The large number of products which appeared on the market solidly support a point to point packet switching technology based on the use of optical fibres.

The emphasis on optical fibre transmission has carried Gigabit Ethernet into the metropolitan area network business, which has been one of the most exciting applications for the new technology. Running over single mode fibre the standard supports distances of up to $5 \mathrm{~km}$. In practice distance spans of tens of kilometres are achievable.

Recently a version of Gigabit Ethernet running over four unshielded twisted pairs has been introduced and products are now on the market supporting this option.

There has been much discussion about the use of so called jumbo frames, frames whose length exceeds that of normal Ethernet frames (roughly speaking up to 9000 bytes instead of 1500). The motivation for using longer frames is to provide higher node throughput when streaming large volumes of data; the fixed frame overhead for transmission and reception being amortized over more bytes. Although significantly higher throughputs are indeed possible using this technique, and products supporting jumbo frames are available from a few manufacturers, they are not part of the standard and not generally supported by the Ethernet equipment suppliers.

\section{OTHER RECENT ADDITIONS TO THE STANDARD}

Two other significant enhancements have recently been added to the 802.3 standard. The first concerns the addition of a virtual LAN tag to a frame to allow a physical LAN to be partitioned into several logical LANs. This facilitates security, privacy and bandwidth management. An additional frame field specifies a user frame priority. This introduces for the first time the concept of quality of service (QoS) within the 802.3 standard, a previous omission pointed out by many of its critics, especially those in the ATM camp.

The second enhancement allows a bundle of parallel links to be aggregated into a single logical link. This so-called trunking standard not only provides increased bandwidth but also offers fault tolerance through redundancy and automatic load redistribution.

\section{TEN GIGABIT ETHERNET}

It seems as if the ink was no sooner dry on the Gigabit Ethernet standard when the next jump in speed was proposed. Indeed a 10 Gigabit task force was set up in March 1999, less than one year after the ratification of the 1 Gigabit standard. The reason for this is quite simply the ever increasing growth of the Internet, doubling every three months, which feeds a demand for ever higher bandwidth. The task force expects to produce a fully ratified standard by September 2002, however, customers can expect to see fully functional pre-standard products early in 2001.

10 Gigabit Ethernet [3], [4] has the following features:

- Standard 802.3 Ethernet frame format and size

- Support for all other legacy features, flow control, trunking, switching etc

- Full duplex only, no CSMA-CD, therefore there is no inherent distance limitation

- Three application spaces: LAN, MAN and WAN

- Only optical fibre is supported

The most significant aspect is the removal of distance restrictions. Only the physics of transmission and the physical media limit the distance spanned by a link. It is this which has opened up the new areas of application. 
The task force has defined a number of alternative optical fibre transmission systems spanning a range of distances from tens of metres up to more than $40 \mathrm{~km}$. It is assumed that eventually the optical components will reach commodity status in terms of price and availability and that for shorter distances the physical layer components will be available a very low cost. Silicon manufacturers are also well advanced with plans for 10 Gigabit support chips.

The use of the new standard over MAN distances will centre on its use directly over a rented (dark) fibre or wavelength. Wide area networking will be facilitated by the provision of an alternative Ethernet WAN encoding system compatible with the existing and widely used SONET/SDH schemes.

\section{PRODUCTS PRICES AND THE COMPETITION}

Ethernet is the clear winner in the LAN field. It is expected to have over $80 \%$ of a $\$ 37 \mathrm{~B}$ LAN equipment market in the year 2000. This market is growing at $20 \%$ per annum. There are very large numbers of firms shipping a great variety of products. Large volumes and intense competition have combined to force prices down. Figure 1 shows the general price trend with time of $100 \mathrm{Mbps}$ and $1 \mathrm{Gbps}$ Ethernet switch ports and network interfaces. It should be noted that there is a price spread at any point in time: in particular, switch port prices depend on the number of ports and the features offered.

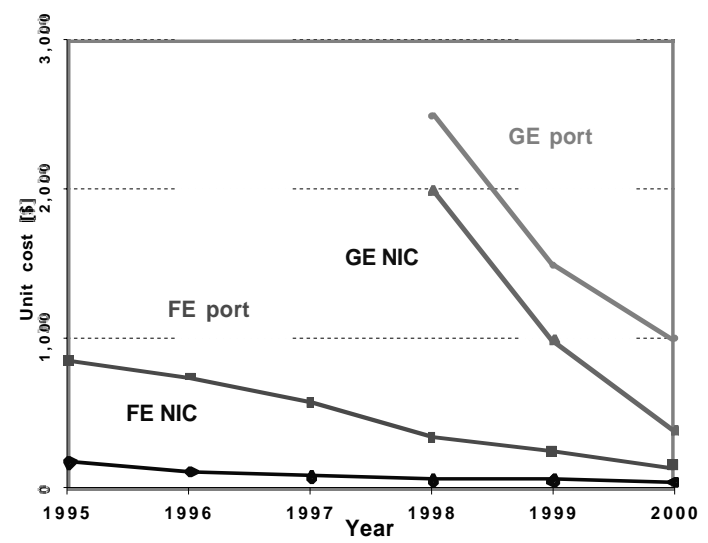

Figure 1: The price of Fast and Gigabit NICs and switch ports as a function of time.

Each new generation of Ethernet traditionally provides ten times the bandwidth at three to four times the cost of the previous generation. Following introduction, prices decrease sharply as both competition and volumes of equipment shipped increase. Figure 2 shows the past and predicted future market trend for the number of Ethernet switch ports world wide. The sharp increases in shipments of 1 and 10 Gigabit systems should be noted.

Since the slogan seems to be "Ethernet everywhere you can use it", it is appropriate to ask whatever happened to the competition? When Ethernet was first standardized in the early 1980 s there was potentially strong competition from IEEE 802 Token Ring and Token Bus standards. Token Ring, strongly supported of course by IBM, did gain some market share but never achieved the universal acceptance of Ethernet.
There do not seem to be any plans to continue its development. As for Token Bus, it simply never made it.

ATM arrived on the scene heralded as the single standard to span the market needs from LAN to MAN to WAN. Many people, including it seems one of the inventors of Ethernet, considered ATM as the "grand unifier". The trouble was it never caught on at all at the desk top. Ethernet was incredibly simple, cheap and robust. It was cheap, it was established and it simply shut out ATM in the LAN market. In the WAN field ATM has of course had considerable success. However, the introduction of Gigabit and especially now 10 Gigabit Ethernet has lead to 802.3 penetration into ATM's traditional areas of application. Some people feel the future could well be just IP packets sent over Ethernet and a very high performance WDM (wave division multiplexing) optical backbone.



Figure 2: The total, world wide, number of switch ports for 10 Mbps, $100 \mathrm{Mbps}, 1 \mathrm{Gbps}$ and $10 \mathrm{Gbps}$ as a function of time.

\section{A SUMARY OF THE PRESENT SITUATION}

Ethernet has evolved from a shared medium LAN running at $10 \mathrm{Mbps}$ to a network based on full duplex flow controlled links running at speeds of up to $1 \mathrm{Gbps}$. Freed from CSMA$\mathrm{CD}$ distance constraints it is able to span ever longer distances. Development of the new 10 Gbps version of the standard is emphasizing long distance operation and smooth integration into the existing WAN world.

There is an enormous choice of Ethernet products and suppliers. This includes a wide range of chip level components as well as hubs, switches, routers and network interface cards. Prices are decreasing steadily with time, very rapidly for Gigabit Ethernet items. The attractive price structure is fuelled by intense competition in a commodity market. Ethernet dominates the LAN scene and this seems unlikely to change in the foreseeable future. A long and healthy future life seems assured.

\section{ETHERNET AT THE LHC}

LHC is scheduled to come into operation in 2005. The purpose of the second part of this paper is to try to project Ethernet into the future on the timescale of the next five to ten years. Two main themes will be explored: Ethernet networks and the attachment of nodes to them. In each case emphasis will be placed on identifying where the limits are likely to be. 


\section{NETWORK TOPOLOGY ISSUES}

A generic picture of a set up for LHC high level triggering is shown in Figure 3. It shows a large number of data sources (buffers) connected to a large number of processors by a network. A representative implementation of the network, using Ethernet switches as basic components, is shown in Figure 4. IEEE 802.3 standards impose a set of rules on what topologies may be used to construct a network. In order to assure all processors can access all buffers a star configuration must be used, with a single high capacity switch at its centre. Figure 4 uses Fast Ethernet to connect the buffers and processors with Gigabit up-links to the central switch. The total Fast Ethernet bandwidth should be in balance with that of the Gigabit up-links.

\section{Data buffers containing events}

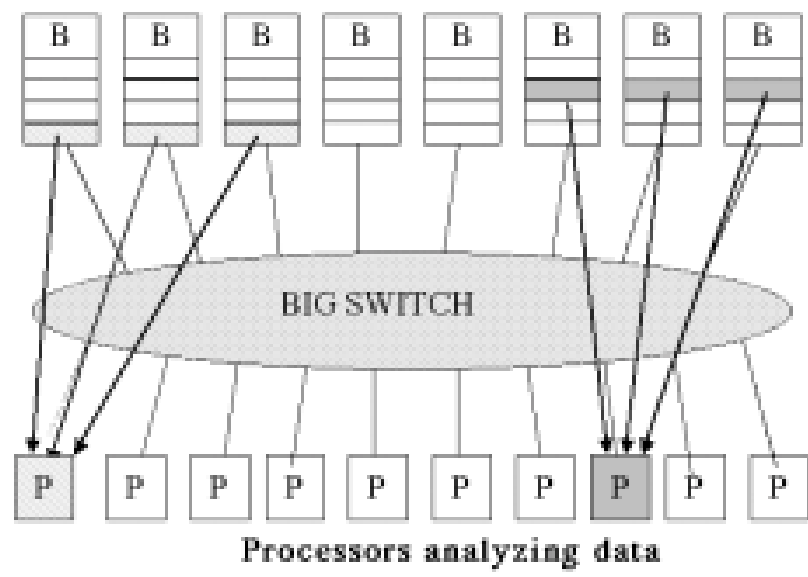

Figure 3: A generic set up for LHC higher level triggering

Provision of the outer layers of switches does not seem to be a problem. Switches with several tens of nodes running at $100 \mathrm{Mbps}$ or $1 \mathrm{Gbps}$ are readily available. Per port prices in general will decrease, in particular for Gigabit switches, and switch sizes will grow. Single chip solutions are becoming available for Gigabit Ethernet switches with 8 to 16 ports. This should lead to end user prices of about $\$ 200$ per Gigabit port.

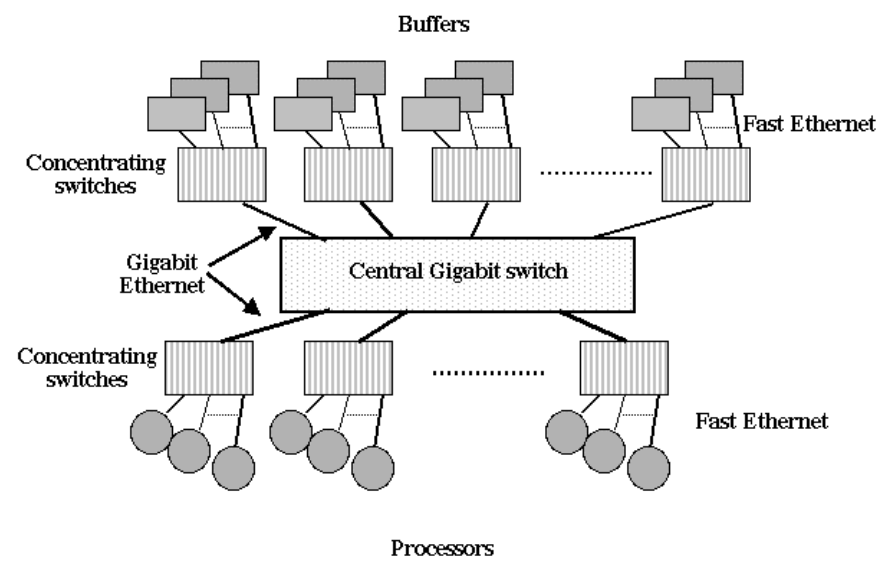

Figure 4: An example of a practical implementation of Figure 3 using Ethernet
Everything comes down to what capacity central switch one will be able to buy on what time scale. This will be the heart of the matter whether the outer switches use Fast Ethernet or Gigabit Ethernet and whether the up links are 1 Gbps or 10 Gbps.

\section{BUILDING HIGH CAPACITY NETWORKS}

Because of the importance of the central switch in Figure 4 we consider in some detail the issues in designing and building such a switch. Figure 5 shows the basic architecture of a large, high performance, Ethernet switch. The physical medium block (PHY) receives frames from the transmission medium and passes them on to the MAC (medium access control). Frames are classified, queued and managed by the network processor (NP) and passed over an adapter (ADAPT), through the switching fabric, to the exit port. The frame reception, processing and buffering, whilst a clear challenge, appears well in hand. Large companies as well as several start ups are addressing these issues. Chips with high performance and functionality are now available at $1 \mathrm{Gbps}$ and the development of $10 \mathrm{Gbps}$ components is underway. The pivotal issue is what capacity switching fabric can be built? This in turn depends on the performance and characteristics of the individual cross bars used in its construction and how they are interconnected to form the overall fabric. The critical parameters are the valency (number of ports) and link speed of the cross bar chip.

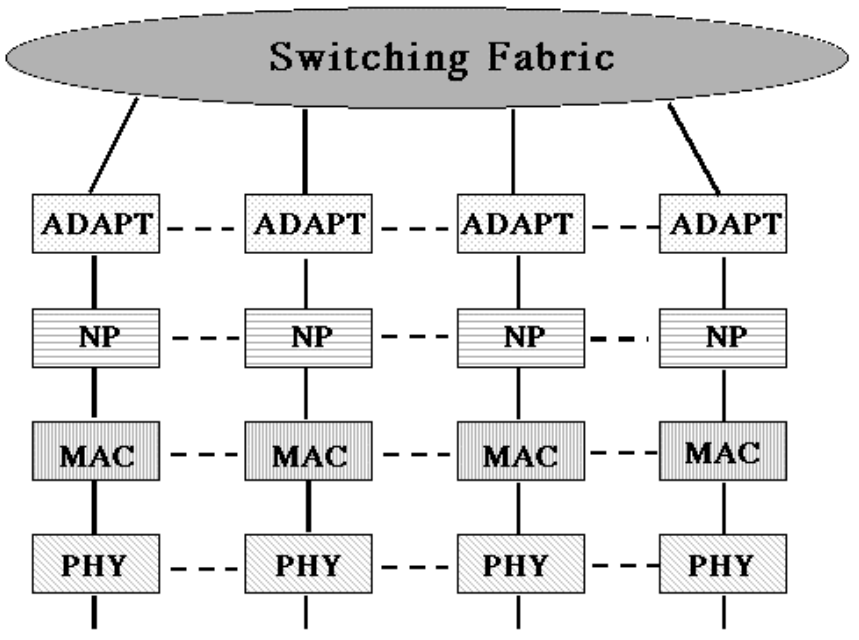

Figure 5: Architecture of a large Ethernet switch

Chips shipping within the next year will have a valency of 32 and a link speed of 2.5 Gbps. This will yield switching fabrics with a throughput in excess of 1 Tbps (Terabit per second). One would expect to see 256/512 port Gigabit switches within the next two to three years. This is a reasonable extrapolation of the largest switches available today, typically 64 ports. On a longer time scale, silicon manufacturers are planning electronic switching fabrics with throughputs well in excess of $10 \mathrm{Tbps}$, corresponding to hundreds of 10 Gigabit ports.

The problem of building very high capacity switches is not simply a question of component chip availability; there are major engineering problems in dealing with a large number of 
very high speed electronic signals at the system level: printed circuit boards, connectors and cables. Optical solutions to these problems are being actively pursued. However, the prognosis for very high capacity switches in the next five years appears very good. They will be needed to keep pace with the ever increasing needs on the Internet.

\section{CHARACTERIZING SWITCHES AND MODELING LARGE NETWORKS}

Computer modeling is a useful tool in the design and optimization of large Ethernet networks and is the subject of an accompanying paper. The approach taken is to test and characterize individual switches and from this information to produce models of the switches that can be used as components in complete network simulations. This allows the performance of different network configurations to be investigated in term of latency and throughput for higher level trigger data collection and processing. The internal structure and details of switching products may affect their suitability for use in LHC applications. This information is not always easy to obtain from manufacturers.

\section{EVENT FARMS IN MEMBER STATES}

Enormous amounts of data may be transmitted over optical fibres. Single fibre capacity is quoted by a well known supplier of world wide optical fibre connections, to be $1 \mathrm{Tbps}$ in 2002, rising to $10 \mathrm{Tbps}$ in 2006. Recent trials, carried out by this company, in collaboration with telecommunications and switching firms, have demonstrated long distance operation of four wavelengths each carrying data at $40 \mathrm{Gbps}$. This included an IP packet stream at 10 Gbps.

Optical fibre networks offer tremendous potential as the backbone of the next generation Internet and will support high performance distributed computing. Despite the present high cost of renting bandwidth, prices are expected to fall in future at about $50 \%$ a year. On the time scale of serious LHC data taking, we would predict a fall in bandwith prices of a factor approaching one hundred. This raises the real possibility of resources in home institutes being used in LHC data taking.

An interesting additional possibility arises for having LHC real time data delivered to a PC in the comfort of your home, in the form of $10 \mathrm{Mbps}$ Ethernet frames, transmitted over your existing phone line, using VDSL techniques (very high bit rate digital subscriber loop). Products for this are now being shipped.

\section{OPTICAL SWITCHING}

There has been much discussion, and more than a little hype, over the question of optical switching. Optical switching does have an important role to play in constructing next generation optical backbones. However, optical switching in this context means circuit switching not packet switching. Various optical devices have been developed to allow point to point optical connections to be dynamically configured. They will appear in products over the next few years. Their advantage is that there will be no need, as now, to go through expensive and bandwidth restricted electrical conversions between the two end points. The typical time to configure or reconfigure a connection is tens of $\mathrm{ms}$.

Given the immense data carrying capacity of optical fibres it is interesting to speculate on whether one could use all- optical packet switching to achieve very high bandwidth packet switching and thus provide an alternative to the electronic switches discussed previously. The prognosis for this is not good in the short term. Optical cross bars with very fast switching times exist (typically $10 \mathrm{~ns}$ ). However, the absence, even on the horizon, of suitable optical buffering and processing components makes most experts believe that all optical packet switching is at least ten years away. The large memories and sophisticated processing found in today's electronic Ethernet switches have at present no photonic equivalents.

\section{QUALITY OF SERVICE}

An LHC experiment, for example ATLAS, may have several logically different networks; for second and third level triggering, for control and monitoring, etc. When considering whether multiple logical networks should be coalesced into a single all purpose physical network, consideration must be given to allocating different priorities and specified bandwidths to different types of network traffic. This topic is referred to as quality of service (QoS) and is the subject of much study in Ethernet circles and beyond. QoS is a key factor allowing different types of traffic, video, voice, email, file transfer, web access, etc., to coexist harmoniously on the Internet.

The Ethernet standard offers rather few direct mechanisms for implementing differential services. QoS is usually implemented on top of the 802.3 standard. Switches typically implement multiple queues in their input and output port interfaces. Frames can be assigned to different queues based on various criteria including; the priority field within an 802.3 frame, the source or destination address of the frame, or some other identifiable property, including TCP and IP packet fields. The way the different queues are filled and serviced determines the details of the QoS offered.

It should be noted that the concept of QoS does not apply only to the network but also to how frames are handled in the host nodes. What matters ultimately is the application to application QoS, not just what happens in the network

\section{THE NODE TO NETWORK INTERFACING PROBLEM AND SOME POSSIBLE SOLUTIONS}

The load imposed on a network node by an Ethernet connection can be large, in some cases restricting dramatically the bandwidth and CPU cycles available to applications. Current, off the shelf products, mainly use the ubiquitous TCP(UDP)/IP protocol stack. Figure 6 shows the rate at which data can be unidirectionally streamed between two 400 $\mathrm{MHz}$ PCs running the LINUX operating system, as a function of message (frame) size, for Fast and Gigabit Ethernet. Network interface cards (NICs) from Intel and Alteon have been used. Also shown is the theoretical limit. It can be seen that Fast Ethernet line rate can only be approached for large messages, when using UDP. The Gigabit Ethernet situation is worse, even for the largest message sizes, only about a third of the possible bandwidth of $125 \mathrm{Mbytes} / \mathrm{s}$ can be achieved.

There are two basic reasons for these limitations. Firstly, the PC CPU limits performance. Figure 7 shows the CPU load of a PC node generating request response operations (a so called ping pong), via TCP/IP, as a function of the rate at which these are carried out. This corresponds to a measured CPU overhead of about $35 \mu$ s for a short message, send or 
receive. For both Fast and Gigabit Ethernet measurements the CPU would be $100 \%$ occupied at request response rates of above $15 \mathrm{kHz}$. The expected ATLAS level 2 request response rate, $14 \mathrm{kHz}$ per processor, is indicated on Figure 7.

These levels of performance make it difficult to use off-theshelf product network software in demanding LHC applications, for example in the ATLAS second level trigger [5]. Optimized I/O drivers, which minimize memory-tomemory copies, interrupts, context switching and elimination of the TCP/IP stack can produce significant improvements in data and message rates [6], [7]. Results achieved with the MESH system [7], developed by the ATLAS experiment, have been added to Figures 6 and 7 to illustrate this. In addition to a much reduced length-independent, per message overhead,

Figure 7 shows that MESH greatly reduces the fixed per message overheads and also eliminates memory-to-memory copies. In this case the CPU load for a given frame rate is virtually independent of the frame length and therefore the lines for the short and long frames in Figure 7 are overlapping.

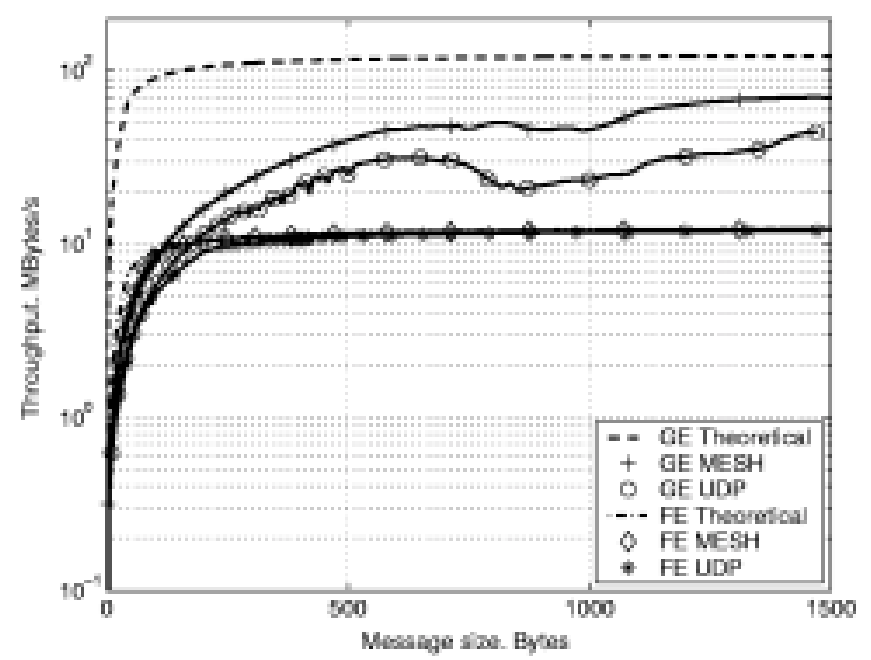

Figure 6: The achieved unidirectional data throughput between two $400 \mathrm{MHz}$ PCs running LINUX.

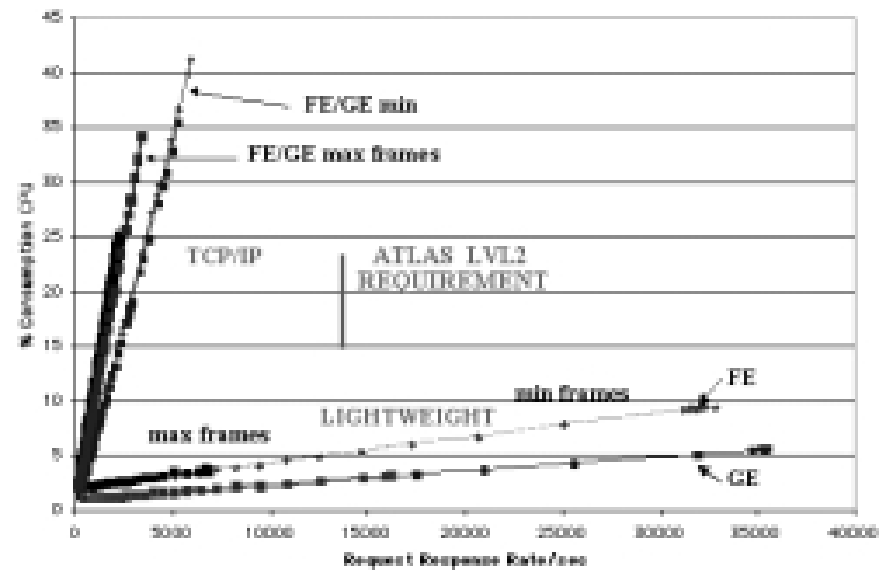

Figure 7: The CPU load on a $400 \mathrm{MHz}$ PC running LINUX as a function of request/response rate, for Fast and Gigabit Ethernet, using TCP/IP and MESH.

For Gigabit Ethernet the PCs PCI bus bandwidth (33 $\mathrm{MHz}, 32$ bit) always strongly limits performance, even for unidirectional transfers. The effect of the PCI bottleneck for
Gigabit Ethernet can be further illustrated by noting that, programming the on board microprocessors used in the Alteon Gigabit NIC to directly generate Ethernet frames, results in line speed being achieved, for all frame sizes and bi-directional operation. This has proved important in characterizing Gigabit switch performance under full load. The greater availability of wider and faster PCI bus versions (66 MHz, 64 bit) will help remove bus bottlenecks, as will the new Infiniband I/O architecture being developed by a significant industry consortium, including, Microsoft, Intel, SUN, Compaq and IBM.

\section{THE NETWORK PROTOCOL ISSUE}

The use of standard Ethernet hardware products is very attractive. However, for high applications where high rates are required, there are often severe drawbacks in using current implementations of TCP/IP, as has been shown previously. This is true whether TCP/IP is implemented over Ethernet or some other network. A common approach that has been adopted, for example in the ATLAS second level trigger studies, is to use optimized I/O drivers and raw Ethernet frames to carry messages. Error rates have been found to be extremely small in practice, allowing a request response mechanism, with a simple time out and retry, to be implemented

The prognosis for using TCP/IP in future remains for the moment unclear. TCP/IP performance surely needs to improve if Gigabit bandwidths are to be fully exploited. Possible improvements are likely to be based on some form of specialized processing at the NIC level combined with more efficient implementations of the host software. Alternative protocols the TCP/IP are also being considered.

\section{SPECIALIZED PROCESSING FOR ETHERNET NETWORKING}

The requirements for very high performance networking are currently being addressed by industry. The dramatic increase in the growth of the Internet must be satisfied and the demands for ever higher performance switches and servers met. A new generation of microprocessor chip is therefore being developed to deal specifically with handling network traffic. These so called network processors have architectures which target I/O and packet processing. They have very high computational ability, in excess of 1 Gips, and usually provide a high degree of integration including several network interfaces.

Network processors will be an important factor in next generation Ethernet switches and routers. They also offer a possible use in NICs, to off load more and more of the networking load from the main host processor (or processors). One might expect to see programmable NICs used to collect data in LHC on-line systems.

Network processors provide a very interesting building block for custom built LHC electronics, in areas where high performance data movement is required. 


\section{CONCLUSIONS}

We conclude by offering a number of predictions which we expect to be fulfilled on the timescale of LHC start up:

- A Gigabit Ethernet connection will be available on your office desk at a per connection price of \$250 (NIC plus switch port).

- NICs will evolve into much more autonomous devices capable of removing most of the communications CPU load from a host processor.

- Links to the home will be at least $10 \mathrm{Mbps}$, at a line rental cost of $\$ 25$ per month.

- Ethernet commodity hardware will be used everywhere in LHC data acquisition and triggering except the front end.

- Public networks are unlikely to meet the needs of HEP. Therefore we will be renting fibres or wavelengths for use between CERN and member states.

- Member state computer centres could provide significant on-line capacity.

\section{REFERENCES}

[1] R. M. Metcalfe and D. R. Boggs, Ethernet: distributed packet switching for local computer networks, available at http://www.acm.org/classics/apr96/
[2] There are a very large number of books, articles and documents about Ethernet, including of course the formal IEEE 802.3 standards. However, no comprehensive bibliography is attempted in this paper. The following two books provide readable overviews of the subject with emphasis on more recent developments: Rich Seifert, Gigagbit Ethernet, Addison Wesley, 1998, ISBN 0-20118553-9 and Stephen Saunders, Gigabit Ethernet Handbook, McGraw-Hill, 1998, ISBN 0-07-057971-7

[3] http://www.10gigabit-ethernet.com/, 10 Gigabit Ethernet Resource Site.

[4] http://www.10gea.org/, Ten Gigabit Ethernet Alliance.

[5] F.J. Wickens et al, The use of commodity products in the ATLAS level-2 trigger, Proceedings of the International Conference on Computing in High-energy Physics, CHEP 2000, Padova, Italy, Feb 2000.

[6] E. Speight et al, Realizing the performance of the virtual interface architecture, International Conference on Supercomputing, Rhodes, Greece, June 1999. See also references cited therein.

[7] M.Boosten et al, High bandwidth concurrent processing on commodity platforms, IEEE Real-Time 99, Santa Fe, USA, June 1999. 